\title{
The coherence of an engineered world
}

\author{
D. Halsmer ${ }^{1}$, J. Asper ${ }^{2}$, N. Roman ${ }^{2} \&$ T. Todd ${ }^{2}$ \\ ${ }^{1}$ Dean, Science \& Engineering, Oral Roberts University, Tulsa, OK, USA \\ ${ }^{2}$ Undergraduate Honors Students of the Department of Engineering \\ \& Physics, Oral Roberts University, Tulsa, OK, USA
}

\begin{abstract}
Normally, information from scientific discoveries is funnelled into the development of engineered products that benefit humanity. But recently a strange turnabout in the flow of practical information has occurred. Concepts from the field of engineering have been found extremely useful in areas of science. From the very large aspects of the universe (i.e. big bang cosmology and galactic and stellar evolution) to the very small aspects (i.e. the fitness of the chemical elements and the coding of DNA for life), the cosmos is so readily and profitably reverse-engineered by scientists and engineers as to make a compelling argument that it was engineered in the first place. The linking of extraordinarily complex, but stable functional structures with the production of value provides the strong impression of a calculating intentionality, which is able to operate in a transcendent fashion. The most coherent view of the universe is that of a system of subsystems that efficiently interact to prepare for, develop, and support advanced life, subject to various physical constraints. The quest for understanding our universe as a whole benefits from the integration of knowledge from all areas of study, including those that consider questions of purpose, such as design engineering. The synthesis of this knowledge that provides the most satisfying answers regarding human experience is one that admits the recognition of purpose and the existence of an (as yet, not-wellunderstood) engineering influence.
\end{abstract}

Keywords: cosmology, reverse-engineering, anthropic principle, engineering.

\section{Introduction}

Throughout the ages, many great minds have expressed a profound appreciation for the incredible ingenuity of natural systems. Leonardo da Vinci, from his in- 
depth study of human anatomy, recorded in his notebook that "The human foot is a masterpiece of engineering and a work of art". He recognized that the intricate structures of the foot provide a synergy resulting in robust functionality without compromising aesthetic value. In a much more recent work, distinguished physicist Walter Thirring writes in Cosmic Impressions: Traces of God in the Laws of Nature, "Chemical forces are able to create the most astounding things out of atoms as if by magic. Life continues this process and takes it to the extreme." Although science has progressed significantly since the days of da Vinci, there remains much to explain regarding the origin of such stable functional structures as the universe, galaxies, solar systems, atoms, and living organisms. The very laws of nature appear to work magically for the benefit of life. Perhaps in the face of such wonder and awe, it is appropriate to apply science writer Arthur C. Clarke's Third Law: "Any sufficiently advanced technology is indistinguishable from magic." Could it be that what we call "nature" is actually advanced technology resulting from a transcendent engineering influence?

Human-engineered systems are characterized by stability, predictability, reliability, transparency, controllability, efficiency, and (ideally) optimality. These features are also prevalent throughout the natural systems that make up the cosmos. However, the level of engineering appears to be far above and beyond, or transcendent of, current human capabilities. Even so, there is a curious match between the comprehensibility of the universe and the ability of mankind to comprehend it. This unexplained matching is a prerequisite for any kind of reverse-engineering activity to be even remotely successful. And yet, mankind seems to be drawn onward toward a potential wisdom, almost in tutorial fashion, by the puzzles of nature that are continually waiting to be unraveled. Indeed, the universe is so readily and profitably reverse-engineered as to make a compelling argument that it was engineered in the first place, apparently with humanity in mind. The fact that the universe appears to be in an unfinished state does not seem to detract from its functionality in providing for and sustaining life. Various aspects of the cosmos are discussed in what follows in an effort to investigate the idea of a transcendently engineered universe.

\section{The engineering of chemistry and physics}

When discussing the origins of the universe, a common question is, "Why is there something rather than nothing?" One should not stop there. Explaining matter is one thing, but explaining matter's behavior is much more difficult. Science has come a long way in discovering the workings of the cosmos, but in some ways the growing confidence in science has become blinding. For example, Newton wondered why objects fall. Eventually, supposedly after seeing an apple fall, he developed the theory of gravity and his three laws of motion. Now, when asked why apples fall, most would be content to say that gravity is the cause. This answer is basically correct, but what is gravity? Why do masses attract each other? Why does matter or energy behave in any orderly manner at all? These are the questions that lead to the idea that the universe may 
be much more than matter and energy. There is a clear difference between describing and explaining. Something much more fundamental must be explained if a more complete understanding of the cosmos is to be achieved. Perhaps this is an area where engineers can play a part by helping to reverseengineer nature's fundamental behavior and meaning.

Author Roy Abraham Varghese refers to the predictable behavior of matter as "embedded intelligence." He defines it as such: "Embedded intelligence describes intelligent systems like atoms that process information by following certain laws, but are not autonomous" [1]. Intelligent systems receive input, process the input, and respond accordingly. There is no computer chip in a force field, yet this process still occurs.

Every great scientific mind has understood in his or her own way that there is great "embedded intelligence" superimposed on matter and energy. Albert Einstein is quoted as saying, "The most incomprehensible thing about the world is that it is comprehensible." Indeed, the governing laws seem to display beautiful unity, symmetry and simplicity, making comprehension and prediction possible. Similarly, Paul Dirac felt awe for the intricate mathematical nature of physics: "God is a mathematician of a very high order and He used advanced mathematics in constructing the universe" [2].

\subsection{The search for the theory of everything}

Some researchers hope to find a natural cause for the orderly laws underlying the universe. The search for a Theory of Everything (TOE) might be such an example. The ultimate goal of a TOE is to reduce the behavior of every event to a single function with one universal constant. Considering the large number of accepted universal constants, this is very ambitious. This universal constant could be used as the variable to model the behavior of other universes if one assumes the existence of a multiverse. Such fields of research may lead to valuable discoveries, but it would be vain to expect to explain the cause of every law in naturalistic terms. As was written above, to explain is much more difficult than to describe. A Theory of Everything may describe the output of a situation given an input, as mathematical functions do. However, it can never explain its own order, nor why what exists interacts in any manner at all.

Where some multiverse concepts fall short is that they merely postulate an infinite set of combinations of the universal constants without considering the actual equations represented. Even if all constants in this universe were reduced to a single TOE with just one universal constant, there is no reason why another universe should obey the same TOE. Otherwise, one would have to explain the origin of the order given by the TOE. Others have recognized the inadequacy of such explanations and have actually proposed that universes with very different behavior may exist. For example, Tegmark proposes a universe obeying mathematics based on fractals or non-Cantorian sets [3]. Paul Davies suggests that some universes might not even obey mathematics, but instead obey purely teleological principles [3]. Do the possibilities stop? What would be the cause of such a strange variety of universes in which mankind is just one infinitesimal occasion? The situation quickly deteriorates into absurdity with no possible 
scientific test to determine truth from fairy tale. In the end, the assumption of a multiverse is purely hypothetical and seems to be proposed simply out of an attempt to reconcile the apparent life-supporting behavior of this universe.

\subsection{The "biofriendliness" of the universe}

The universe is undoubtedly "biofriendly." Christian de Duve (Biochemist, Nobel laureate in medicine) states just how obvious this concept has become among the scientific community:

We live in a biofriendly world. Were it otherwise, we wouldn't be around. The question is, therefore, how biofriendly is it? Physicists have addressed this question and have come to the conclusion that if any of the fundamental physical constants were a little smaller or a little larger than they are, the universe would be very different from what it is and unable to produce or harbor living organisms. [4]

So just how biofriendly are the physics and chemistry of the universe? Lawrence J. Henderson wrote a very relevant book to this study in 1913 entitled The Fitness of the Environment. A 1913 review called it a "logical sequel to the Origin of Species" [5]. A tribute to Henderson's work, entitled Fitness of the Cosmos for Life, was just published in 2008. Henderson's overall point is that "Fitness there must be, in environment as well as in the organism" [6]. He claims that first of all, life must be complex - physically, chemically, and physiologically. Also, life and its environment must be durable, especially chemically. Third, it must have metabolism, exchanging energy and matter to maintain an improbable shape [7]. The environment plays a critical role in allowing for the possibility of such an unlikely mechanism as a living organism. Indeed, some even claim that the environment is the engineer that designs life. Let's dig deeper into how chemistry and physics behave to see if this seems feasible, or if the laws of nature actually appear to be engineered themselves.

\subsubsection{The incredible properties of water and carbonic acid}

Incredibly, most of Henderson's observations are still very important today despite writing Fitness before Rutherford's theory of the nuclear atom had been applied. Henderson primarily focuses on the life-supporting properties of carbonic acid $\left(\mathrm{H}_{2} \mathrm{CO}_{3}\right)$ and water. Carbonic acid is to be thanked for the constancy of the alkalinity of both blood and the oceans. The common but vital reaction of carbonic acid is fairly simple $\left(\mathrm{CO}_{2}+\mathrm{H}_{2} \mathrm{O} \rightarrow \mathrm{H}_{2} \mathrm{CO}_{3} \rightarrow \mathrm{H}^{+}+\mathrm{HCO}_{3}{ }^{-}\right)$. This reaction can go either direction, forming a stable system. It acts as a great buffer against a fluctuating acidity by allowing excess acid to automatically be carried by the blood and then out of the lungs. Henderson states that the chemist cannot rival its efficiency [12]. Michael Denton describes it as a "solution of breathtaking elegance and parsimony" [13].

The remarkable properties of water are numerous. Its very high specific heat maintains relatively stable temperatures both in oceans and organisms. As a liquid, its thermal conductivity is four times any other common liquid, which makes it possible for cells to efficiently distribute heat. On the other hand, ice has a low thermal conductivity, making it a good thermal shield in high latitudes. 
A latent heat of fusion only surpassed by that of ammonia tends to keep water in liquid form and creates a natural thermostat at $0^{\circ} \mathrm{C}$. Likewise, the highest latent heat of vaporization of any substance - more than five times the energy required to heat the same amount of water from $0^{\circ} \mathrm{C}$ to $100^{\circ} \mathrm{C}-$ allows water vapor to store large amounts of heat in the atmosphere. This very high latent heat of vaporization is also vital biologically because at body temperature or above, the only way for a person to dissipate heat is to sweat it off [8].

Water's remarkable capabilities are definitely not only thermal. A high vapor tension allows air to hold more moisture, which enables precipitation. Water's great surface tension is necessary for good capillary effect for tall plants, and it allows soil to hold more water. Water's low viscosity makes it possible for blood to flow through small capillaries. A very well-documented anomaly is that water expands into the solid state, which keeps ice on the surface of the oceans instead of accumulating on the ocean floor. Possibly the most important trait of water is its unrivaled solvency abilities, which let it transport great amounts of minerals to immobile organisms and also hold all of the contents of blood. It is also only mildly reactive, which keeps it from harmfully reacting as it dissolves substances [8]. Recent research has gone into understanding how water acts as an efficient lubricator in many biological systems from snails to human digestion. By itself, water is not very effective in this role, but it works well with certain additives, such as some glycoproteins [9]. The sum of these traits makes water an ideal medium for life. Literally every property of water is suited for supporting life. It is no wonder why liquid water is the first requirement in the search for extraterrestrial intelligence.

All of these traits are contained in a simple molecule of only three atoms. One of the most difficult tasks for an engineer is to design for multiple criteria at once. As engineering professor Henry Petroski notes, "All design involves conflicting objectives and hence compromise, and the best designs will always be those that come up with the best compromise" [10]. Satisfying all of these criteria in one simple design is an engineering marvel. Also, the design process goes very deep, since many characteristics would necessarily be changed if one were to alter fundamental physical properties such as the strong nuclear force or the size of the electron. Paul Davies comments on the subject:

It happens that water combines in one substance several key qualities (thermal, mechanical, chemical) that life exploits and indeed that are indispensable to life as we know it. What are we to make of this? Is it just a lucky fluke that the same stuff that has an anomalous expansion property when it freezes (enabling ice to float) also has superlative solvent properties or unusually high surface tension and/or efficient tectonic lubrication qualities, for example? [3]

The molecular shape of water explains most of its incredible thermal properties. The molecule is a triangle at a 104'30' angle. The electron of each hydrogen atom is pulled towards the oxygen atom, exposing the positive proton nucleus of the hydrogen atoms a bit. The nucleus of the oxygen atom is overcompensated, leaving its exposed shoulder with a negative charge. The water molecules are therefore able to become weakly coupled and re-coupled (a 
hydrogen bond). The lower density of ice is because the crystal structure opens up and the coupling effect is reduced in ice. Henderson did not know about hydrogen bonds, but they play other vital roles in organisms. For example, the strands of DNA are joined by hydrogen bonds, which are strong enough to hold together but weak enough to unzip when it is time for replication, a very beautiful design [11].

\subsubsection{Hydrogen, oxygen and carbon}

Conveniently, the three elements in water and carbonic acid are the same ones which have the ability to create compounds with the incredible amount of variety and complexity necessary for life. Henderson comments on this:

That the very elements which make up water and carbonic acid, and apparently they alone, should posses this wonderful property [complexity, variety] is, when taken together with the physical properties of water and carbonic acid and their place in cosmic evolution as constituents of the atmosphere, a fact which cannot lightly be set aside. [14]

Henderson could not have known why these elements occur in such abundance. Hydrogen, oxygen and carbon rank one, three and four in prevalence in the universe (helium is the other). The explanation has to do with fusion within stars. Early reactions start with hydrogen atoms and then produce deuterium (mass 2), tritium (mass 3), and alpha particles (mass 4), but no stable mass 5 exists. This limits the creation of heavy elements and was considered one of "God's mistakes" until further investigation. In actuality, the lack of a stable mass 5 necessitates bigger jumps of four which lead to carbon (mass 12) and oxygen (mass 16). Otherwise, the reactions would have climbed right up the periodic table in mass steps of one (until iron, which is the cutoff above which fusion requires energy rather than creating it). The process would have left oxygen and carbon no more abundant than any other element.

One might wonder why Beryllium (mass 8) is not more prevalent. This is because it too readily reacts with helium to create carbon. Stability is an important consideration here, and it is also a very important feature of most engineered systems. Energy resonance levels are the major determinant of stability. Resonance here refers to how beryllium and helium have a combined energy almost exactly equal to an excited carbon atom. Cosmologist Fred Hoyle actually predicted carbon's resonance level based solely on the anthropic assumption that it was necessary in large quantities for life. He was later proven correct. If the resonance level of carbon was $4 \%$ lower, essentially no carbon would form. If it was $0.5 \%$ higher, almost all carbon would react and become oxygen [11].

An interesting engineering analogy here would be LEGOs ${ }^{\circledR}$. The reason why LEGOs are so useful and fun is because they stay together when assembled into an object. On the other hand, they also can be broken down and reassembled when desired. In order to make this possible, engineers in Denmark had to carefully design the perfect interference fit. If the fit is too tight, the pieces will never attach or never come apart, while if it is too loose, they will never stay together. LEGOs are actually manufactured to a tolerance of $2 \mu \mathrm{m}$ [15]. In the 
same way, if the "fit" of carbon had been 4\% "tighter," it would have rarely ever formed, and if it had been $0.5 \%$ "looser," it would have rarely stayed together.

Though he remained agnostic, Hoyle's prediction of carbon's resonance level must have surprised even him, because it led to some interesting quotes:

A commonsense interpretation of the facts suggests that a superintellect has monkeyed with physics, as well as with chemistry and biology, and that there are no blind forces worth speaking about in nature. [16]

And also:

I do not believe that any scientist who examined the evidence would fail to draw the inference that the laws of nuclear physics have been deliberately designed with regard to the consequences they produce inside the stars. [16]

\subsubsection{Physics}

Physics, and not just chemistry, has played a part in the examples so far, but it has not yet been discussed directly. Several people have made lists of physical values that must be "fine-tuned" for life to exist as it does. Astronomer Hugh Ross lists 154 such examples on his website [17]. These optimalities suggest the influence of a calculating intentionality or some kind of transcendent cosmic engineer. Three instances of different types of fine-tuning of physics are below [18]:

1) The ratio of gravity to the electromagnetic force is nearly perfect for star variety and therefore element variety and also for long life spans of stars (the preciseness is on the order of $1: 10^{40}$ ).

2) The ratio of electron mass to proton mass allows for long chains such as DNA.

3) Three dimensions are the only possible dimensional space that allows for stable orbits, which are needed for atoms and solar systems.

Many more inferences of engineering can be drawn from physics. Some will be examined more closely in the section on cosmology.

\subsection{Beauty}

The concept of beauty is important in engineering. In most cases, it has been used above to describe a beautiful solution. Generally, in terms of engineering, a beautiful solution is one that optimally fulfills many constraints in the simplest manner possible. Water is an excellent example. Beauty is also something that can be superimposed over a design with no apparent usefulness other than pure enjoyment. This concept is significant in the automotive industry, for example. It is difficult to deny that nature also contains immense beauty. An example that satisfies both of these types of beauty would be light. Light is useful for energy transfer and information transfer and is the speed limit of the universe, yet it also exhibits the beautiful array of colors that the human eye can perceive.

There are many reasons to suspect that chemistry and physics show signs of being engineered at a transcendent level. These can be found from the subatomic level all the way up to the cosmic level. Currently, the most common way to refute this is not to deny that the behavior of the universe is improbably convenient for life, but to claim that there are nearly infinite other universes that 
were not so fortunate. This solution, however, makes very large assumptions and still must answer some of the crucial causal questions regarding matter and its behavior. Considering this, the hypothesis of a transcendent engineer may not be as improbable as it might originally seem.

\section{The engineering of the cosmos}

The universe is a multifaceted complex system with countless far-reaching parts and subsystems. It is so vast that it is impossible for man to measure and comprehend all of its secrets, but what we can do is extrapolate from what we are able to measure and observe. By viewing the universe as a transcendently engineered system, we are able to apply the principles of reverse engineering to glean some aspects of the meaning and purpose of the universe.

\subsection{Product teardown}

One of the main steps in reverse engineering is the product teardown. Product teardown is the process of taking apart a product to understand it and to understand how the company making the product succeeds [19]. Applying this method to the universe allows us to think about "dismantling" it into its various galaxies, planets, and other subsystems in order to see why it works the way it does.

Galaxies fall into three basic types: spirals, ellipticals, and irregulars. The best galaxies for observation come from what is known as "The Local Group" coined by Edwin Hubble in his book The Realm of the Nebulae. The Local Group refers to the small cluster of stellar systems surrounding our own galaxy. The Local Group contains examples of most major types of galaxies except giant ellipticals. This is actually a good thing because any giant elliptical galaxy even remotely near to our own galaxy would cause enough cosmological damage to prevent life on Earth [20]. The Local Group is just a small portion of the approximately hundred thousand million galaxies that can be seen using modern telescopes, but their proximity has allowed for great developments in the study of galaxy composition and formation.

These galaxies in turn hold about a hundred thousand million stars. Our Sun is an example of an average-sized, yellow star, near the outer edge of the spiral arms of the Milky Way galaxy. Around our Sun are the planets of our solar system. Each one is unique yet contributes to the whole of the system. The most unique being Earth as it is the only habitable planet known by mankind.

The New York Times in a February 2008 article cited that around 250 planets outside of our solar system have been found since 1995 [21]. The majority of these planets were found using the Doppler Detection method, with six of them being found through a new method called microlensing. The new planets could possibly form a solar system that is similar to our own although no life is believed to exist on the discovered planets.

The majority of the systems that have been found vary from ours in two important ways. First, some of the giant planets in these systems orbit very close 
to their host stars with periods as little as three days. Second, the planets with orbital periods longer than a few weeks generally have highly elongated orbits, changing drastically in their distance from host stars. This makes our solar system an oddity in comparison with most of the neighboring systems [22].

Now that we are looking at galaxies and planets as components of the whole universe, we must not forget to include the importance of universal constants and laws in our list. After all, these constants and laws act as our nuts and bolts. Without them there would be no order to hold the universe together for the benefit of life. There are numerous constants that hold throughout the universe. Some of the more widely known constants include vacuum permittivity, vacuum permeability, the constant of gravitation, Planck's constant, and the speed of light in a vacuum. There are also many electromagnetic and chemical constants that hold true throughout the whole universe. Along with these constants are the laws that govern our universe, such as Newton's laws, the laws of Thermodynamics, and Maxwell's equations. All of these play an important role in the life-friendly structure and order of the universe.

\subsection{Functionality}

When it comes to galaxies, the bulk of our knowledge comes from our own galaxy and the galaxies nearest to it. Looking at these galaxies alone, there seems to be no functional reason for existence. The importance becomes apparent when the galaxy is treated in respect to the whole. One of the greatest intellectual revolutions in the twentieth century comes from the discovery that the universe is expanding. The rate of this expansion can be determined by measuring the velocities at which other galaxies are moving away from us using the Doppler Effect [23]. This expansion rate is very specific in that it is based on the density of the universe and the gravitational pull caused by this density. With an increase in universal density on the order of just $1: 10^{60}$ the gravitational pull would cause the universe to collapse on itself, while a density slightly lower by the same amount would cause the universe to expand at such a rate where galaxies would most likely never form at all, let alone contain life [18]. This evidence suggests the influence of a calculating intentionality that engineered the large-scale structure of the to support one or more life-sites.

Planets are important not for the universe as a whole, but as a home base for life. Therefore planets could be called the "customer satisfaction" products of the universe. More than just being habitable itself, a planet can offer protection for other planets. One example of this is the protection Jupiter provides for us from flying debris. The gravitational pull of Jupiter keeps most asteroids, comets, etc. from ever reaching the inner solar system, thus protecting Earth from lifethreatening bombardments [22].

The universal constants keep the order in the universe. These constants ensure predictability and repeatability of what we consider the laws of the universe. For an engineered system, all of these constants must be fine-tuned to ensure a proper working order, and that is what we see in the universe. Giuseppe Del Re summarizes it well: 
First premise: Our present view of the history of the universe is based on the accepted general laws of nature.

Second premise: Those laws involve precise universal constants, i.e. special values of certain physical quantities.

Third premise: If any one of those universal constants had a value slightly different from the one it has, then a) the processes which have produced the chemical elements necessary for life such as we know it would not have taken place, at least within the estimated life of the universe; b) even if they had taken place, evolution wouldn't have had time to produce Homo sapiens. [24]

Now that the functionality of the system is established, the only other necessary parts to engineer are those used to create customer satisfaction. There are many things in this universe that seem to work for our benefit, but they fit mostly into three categories in reference to an engineered system: 1) The "parts" that create the ability for human life; 2) The potential for discovery that allows us to discern the workings of the universe; 3) The aesthetic value of the universe.

\subsection{Requirements for Life}

The requirements for life range from the macroscopic to the microscopic scale. On the universal scale however, one can see that our planet is in a comparatively narrow region of space known as the "Galactic Habitable Zone" [25]. This Zone allows for the right surface temperature, stable climate, metallicity, the ability to hold liquid water, and many other conditions necessary for life. There is no practical reason why the universe has to contain life, but the fact that we are here gives great importance to this zone for the benefit of our existence.

Not only does this zone satisfy the requirements of life, it has also endowed us with a prime position to view the wonders of the universe. There are many qualities that make the Earth an excellent place to study the universe from. First of all is the transparency of the atmosphere. Our atmosphere admits the radiation necessary for life, while blocking most of its lethal energy. This transparency also allows us to see into space without the distortions caused by a thick atmosphere such as Venus. Secondly, the regularity of our solar system's orbits makes time calculation of planetary events more predictable, even allowing for estimations of planetary orbits millions of years ago [22]. Finally, the gas and dust in our region of the Milky Way are diffuse compared to other regions in the local mid-plane. This allows us to view 80 percent of the universe without blockage. If our solar system were moved farther away, perpendicularly to the mid-plane, we would be able to see the other 20 percent. However, this would cause a large percentage of our current view to be blocked by dust as well as the luminosity of stars in close proximity [20].

Our place in the universe is amazingly unique when it comes to discovery. Our planet is in prime position for the gleaning of knowledge from the stars. The only thing that makes our universe more amazing is its breathtaking beauty. Looking up into the night sky, one cannot help but wonder at the vast intricacy of the universe. In the words of Stephen M. Barr:

...[if] the laws of nature are based on symmetries that are so sophisticated and so deep that while we may study them with the tools of modern 
mathematics they lie far above our mental powers to appreciate on an intuitive level, does that not suggest the mind of an artist at work that is far above the level of our own minds? When we contemplate this strange and beautiful universe, well may we ask, in the words of the poet Blake, "What immortal hand or eye could frame thy fearful symmetry?" [26]

From its parts to its whole, the universe demonstrates that it is both precise and planned, strongly suggesting that it is specifically engineered to support life. In addition, this life-support system readily lends itself to the reverse engineering process, which also confirms this perspective, assuming a working knowledge of the cosmos is of value to intelligent beings.

\section{The engineering of life}

A lot of common engineering designs have come from looking at biological systems. A prime example of this is the propeller. However, it is a common error to overlook the importance of the fact that these biological systems exhibit clear evidence of being engineered systems. Biological systems are constantly undergoing processes that exhibit modularity, specificity, adaptability, durability, and many other aspects of engineered systems. In the Design of Life, William Dembski writes that "Many of the systems inside the cell represent nanotechnology at a scale and sophistication that dwarfs human engineering. Moreover, our ability to understand the structure and function of these systems depends directly on our facility with engineering principles." [27]

For the sake of space, we will not break down every biological system and investigate the engineering principles they exhibit; therefore, it is important to note that these principles are not just applicable to the examples put forth, but apply to virtually all biological systems.

\subsection{Construction of biological systems}

All things that are engineered start out as a concept that is designed with the end in mind. What purpose will this serve? What will be needed to construct the functional structure? These are some of the common questions asked when in the first stages of engineering. After these questions have been answered, the next step is to acquire the materials needed and to start building, which in itself is a complex process, because if one thing is out of place, or not attainable, the whole system is compromised. In his book, the Edge of Evolution, Michael Behe refers to this overall process as being a "bottom uptop down" design. He writes, "by bottom up I mean that of course the foundation of the building had to be poured first, the ground floor next, and so on, all the way to the zenith at the sixth floor," and that "by top down I mean that the building was planned" [28]. The construction of a cilium appears to follow the same process. When a cell starts putting out a new cilium, it gathers the materials that it needs to start the process and stores them at its base until they are needed. 


\subsection{Reverse-engineering of biological systems}

When the propeller was first engineered, the developers found the basis of their design by looking at a cell and analyzing a flagellum. Scientists and engineers then used reverse-engineering to discover how the flagellum was able to produce movement of the cell and applied these principles to large-scale systems that we now use for transportation. The state of the flagellum is constantly monitored and maintained. Perhaps one of the best examples of engineering in a biological system is the flagellum, which is:

thought to be the only structure in nature that has a rotary motion, acting as a highly efficient propeller that drives a bacterium forward or backward. It is typically made up of about 50 proteins and builds itself from the inside out. Its precision motor is powered by the movement of protons, with a drive shaft equipped with a cylindrical bushing that pokes through the bacterium's membrane. It is like an outboard boat motor only much faster and more efficient, and if a single part were missing, it clearly would not work. [29]

An interesting feature regarding the construction of the flagellum is that as the final piece of a section is being put in place, it brings a coded gene that initiates the next phase of the construction. This enables the flagellum to be built in an extremely efficient manner.

\subsection{Modularity of life}

Another engineering principle that is being recognized in biological systems more frequently is that of modularity. In his book, System Modeling in Cellular Biology: From Concepts to Nuts and Bolts, Zoltan Szallasi talks about how biological systems show signs of being modular in design.

Almost all artifacts of evolved human engineering are modular through and through: their entire architecture is composed of parts packaged within bigger parts with clear functional interfaces. This is true of electronics, it is true of houses, and it is true of software. In these systems, all parts are members of some module, and the entire architecture is modular. It would seem, based on this experience, that the way forward in simplifying the complex biochemistry of life is to encapsulate complexity in similar modules. [30]

There are two common types of modularity, "function-based" and "manufacturing-based." Cells exhibit both types of modularity. First off, there are many different types of cells within all living creatures that have different functions. Red blood cells contain hemoglobin, which is used to transport oxygen to the various regions of the body. If it also had the function of the white blood cell then pandemonium would ensue. If modularity did not exist, it would be extremely difficult for a system, in this case a cell, to be reconfigured. A group called El-Samad recently researched the bacterium Escherichia coli, or E. coli, viewing it as a modular system.

In a top-down manner within this module, the authors have performed a systematic model reduction, and they have proposed the existence of certain functional submodules based on characteristics of the overall behavior of the entire system, such as robustness... or optimal performance. This approach 
closely follows the method of modular decomposition routinely used in system engineering, namely the identification of submodules or devices based on their dynamic functions. [31]

The ability to breakdown biological systems into modules and submodules is nothing new, since it is recognized that various organs and cells inside the body have modular characteristics. A common example of this is that of organ transplants. If body organs were not modular, it would be nearly, if not completely impossible for transplants to be successful.

\subsection{Adaptability}

Another important principle is that of adaptability. If a system is to last in a changing environment for any period of time, it has to be able to adapt to its surroundings. One of the best examples of biological adaptability exists between malaria and the immune system of humans. Malaria gets into the bloodstream, attaches itself to a red blood cell, travels through the bloodstream until it reaches a prime organ, such as the liver, and then duplicates quickly to infect many more red blood cells. Over the years it has had to continuously adapt to changes of the immune system. Mutations in the cellular structure of the body have brought about certain changes which malaria has had to overcome. The most common of these is known as Sickle Cell disease, characterized by irregularly shaped blood cells, which combat malaria by causing the red blood cells to stick together and sweep the infected red blood cells through to the spleen, which is a very effective filter for old cells.

When we look at the human body, we see that there are millions of complex systems working together to create and sustain growth and maturation. The fact that these cells work so well together is remarkable, especially considering the complexity involved with the DNA strand and genes. The human body, and the bodies of other living organisms as well, are functional systems that clearly exhibit evidence of not only being designed, but of being engineered.

\section{Conclusion}

An interdisciplinary study of the cosmos suggests that a transcendently engineered world may be the most coherent explanation. E.O. Wilson recently revived the term "consilience" in reference to the unity of knowledge; "literally a "jumping together" of knowledge by the linking of facts and fact-based theory across disciplines to create a common groundwork of explanation.' [32] This phenomenon is currently being realized across the physical, life, and social sciences within the context of an engineering mindset. The universe displays a beautiful functionality which seems to automatically deploy for the benefit of life and mankind in particular. Even so, humans are largely able to comprehend the workings of the cosmos, and recognize widespread technological attributes that dovetail into a consilience that is best explained by the wisdom of a transcendent engineer. The idea of transcendence indicates that we detect an engineering capability that is above and beyond the limits of our ordinary experience and 
possibly beyond our material existence. The discerned laws that govern the behavior of matter, energy, and information over space and time display a sublime ingenuity and intentionality that many (especially scientists and engineers) recognize as significant for worldview considerations.

\section{References}

[1] Varghese, R.A., The Wonder of the World, Tyr Publishing: Fountain Hills, p. 27, 2003.

[2] Dirac, P.A.M., The Evolution of the Physicist's Picture of Nature. Scientific American. p. 53, May, 1963.

[3] Davies, P.C.W., Fitness of the cosmic environment (Chapter 6). Fitness of the Cosmos for Life, ed. J.D. Barrow et. al., Cambridge University Press: New York, pp. 100-110, 2008.

[4] de Duve, C., How biofriendly is the universe? (Chapter 10). Fitness of the Cosmos for Life, ed. J.D. Barrow et. al., Cambridge University Press: New York, p. 169, 2008.

[5] Mendelsohn, E., Locating "fitness" and L.J. Henderson (Chapter 1). Fitness of the Cosmos for Life, ed. J.D. Barrow et. al., Cambridge University Press: New York, p. 13, 2008.

[6] Henderson, L.J., The Fitness of the Environment, The MacMillan Company: New York, p. 6, 1913.

[7] Henderson, L.J., The Fitness of the Environment, The MacMillan Company: New York, pp. 267-69, 1913.

[8] Denton, M.J., Nature's Destiny: How the Laws of Biology Reveal Purpose in the Universe, The Free Press: New York, pp. 19-46, 1998.

[9] Tyler, D., The Remarkable Phenomenon of Biological Lubrication; Access Research Network. 11 Feb, 2008. http://www.arn.org/blogs/index.php/ literature/2008/02/11/the remarkable phenomenon of biological

[10] Petroski, H., Invention by Design: How Engineers Get from thought to Thing, Harvard University Press: Cambridge, p. 30, 1996.

[11] Gingerich, O., Revisiting The Fitness of the Environment (Chapter 2). Fitness of the Cosmos for Life, ed. J.D. Barrow et. al., Cambridge University Press: New York, pp. 20-30, 2008.

[12] Henderson, L.J., The Fitness of the Environment, The MacMillan Company: New York, pp. 147, 1913.

[13] Denton, M.J., Nature's Destiny: How the Laws of Biology Reveal Purpose in the Universe, The Free Press: New York, pp. 132-33, 1998.

[14] Henderson, L.J., The Fitness of the Environment, The MacMillan Company: New York, pp. 219-20, 1913.

[15] Company Profile 2007; The LEGO Group. p. 7. http://www.lego.com/info/pdf/LEGO_company profile_UK.pdf

[16] Hoyle, F., The Universe - Past and Present Reflections. Annual Review of Astronomy and Astrophysics. 20, pp. 8-17, 1981. 
[17] Ross, H., Fine-Tuning For Life on Earth; Reasons to Believe, 2004. http://www.reasons.org/resources/apologetics/design_evidences/200406_fi ne tuning for life on earth.shtml

[18] Holder, R.D., Go $\bar{d}$ the Multiverse and Everything, Ashgate Publishing: Burlington, pp. 33-39, 2004.

[19] Otto, K. \& Wood, K., Product Design Techniques in Reverse Engineering and New Product Development, Prentice Hall: Upper Saddle River, p. 135, 2001.

[20] Bergh, S.V.D., The Galaxies of the Local Group, Cambridge University Press: New York, p. 1, 46, 2000.

[21] Overbye, D., "Smaller Version of the Solar System Is Discovered," NY Times. 2008 Feb 15.

[22] Gonzalez, G. \& Richards, J.W., The Privileged Planet, Regnery Publishing: Washington DC, pp. 66, 93-95, 2004.

[23] Hawking, S.W., The Theory of Everything, New Millennium Press: Beverly Hills, p. 33, 2002

[24] Del Re, G., The Cosmic Dance, Templeton Foundation Press: Radnor, p. $173,2000$.

[25] Gonzalez, G.D.B. \& Ward, P.D., “The Galactic Habitable Zone,” Scientific American, p. 60-67, (October 2001).

[26] Barr, S.M. Modern Physics and the Ancient Faith. Sheridan Books, p. 104, 2003.

[27] Dembski, W.A. \& Wells, J, The Design of Life, The Foundation for Thought and Ethics: Dallas, p.256, 2008.

[28] Behe, M,. The Edge of Evolution, Free Press: New York, p. 85, 2007.

[29] Witham, Larry. By Design: Science and the Search for God, Encounter Books, 2002.

[30] Szallasi, Z, System Modeling in Cellular Biology: From Concepts to Nuts and Bolts. MIT Press: Cambridge, p. 48, 2006.

[31] Szallasi, Z, System Modeling in Cellular Biology: From Concepts to Nuts and Bolts. MIT Press: Cambridge, p. 42, 2006.

[32] Wilson, E.O., Consilience: The Unity of Knowledge, Vintage Books, New York, p. 8, 1998. 